QUANTUM PROBABILITY

BANACH CENTER PUBLICATIONS, VOLUME 73

INSTITUTE OF MATHEMATICS

POLISH ACADEMY OF SCIENCES

WARSZAWA 2006

\title{
QUANTUM STOCHASTIC DYNAMICS ON TYPE III FACTORS
}

\author{
P. LUGIEWICZ and ROBERT OLKIEWICZ \\ Institute of Theoretical Physics, Wrockaw University \\ Pl. Maxa Borna 9, 50-204 Wrockaw, Poland \\ E-mail: piotrlug@ift.uni.wroc.pl,rolek@ift.uni.wroc.pl
}

1. Introduction. There have been much interest in the irreversible dynamics of quantum systems in recent years. Most of it, however, concentrated either on finite dimensional systems described by matrix algebras (as for example in quantum optics [1]) or on the standard quantum mechanics [2-6]. In the later case observables of the quantum system are represented by self-adjoint operators acting on some Hilbert space and states are described by positive normalized trace class operators, the so-called density matrices. In both cases a number of the master equations which describe irreversible evolution of the quantum system in the Markovian approximation have been discussed. However, many of realistic physical systems like Bose or Fermi gases cannot be represented by such simple objects. They are described by the GNS representations of some abstract $C^{*}$-algebras. For infinite spin systems, that is for canonical anticommutation relations (CAR) algebras, the concept of the quantum Markov semigroup has been studied in a number of papers, see for example [7-9]. The discussion of irreversible dynamics of canonical commutation relations (CCR) algebras is less advanced and concentrated mainly on the so-called quasi-free dynamical semigroups $[10,11]$. They are, however, interesting objects for physicists since type III factors being the $\sigma$-weak closure of the temperature representations of the CCR algebras describe realistic Bose gases. In this paper we address the question how one can construct a class of quasi-free dynamical semigroups both on the CCR algebras and the corresponding von Neumann algebras.

2. Algebras of canonical commutation relations. The aim of this section is to set up a formalism in which we can discuss systematically the canonical commutation relations for a finite and infinite number of degrees of freedom. A comprehensive discussion of this subject can be found in [12]. Suppose $S$ is a real linear space equipped with a

2000 Mathematics Subject Classification: 46L57, 47D06.

The paper is in final form and no version of it will be published elsewhere. 
nondegenerate symplectic bilinear form $\sigma: S \times S \rightarrow \mathbf{R}$. Moreover, we assume that there exists a linear operator $J$ on $S$ with the following properties

$$
\sigma(J f, g)=-\sigma(f, J g), \quad J^{2}=-\mathrm{id} .
$$

By means of $J$ and $\sigma$ one can introduce a complex pre-Hilbert space with the scalar multiplication and the scalar product defined by

$$
\begin{gathered}
\left(\lambda_{1}+i \lambda_{2}\right) f=\lambda_{1} f+\lambda_{2} J f, \quad \lambda_{i} \in \mathbf{R}, \\
\langle f, g\rangle=\sigma(f, J g)+i \sigma(f, g),
\end{gathered}
$$

where $f, g \in S$. Its norm completion will be denoted by $\mathcal{H}$. On $S$ there is usually defined its own topology $\tau$, which is stronger than the norm topology and which makes $S$ a real locally convex topological vector space, for the definition see the next subsection. The case in which $S$ is infinite dimensional is typical for field theories and many-body problems, whereas finite dimensional $S$ corresponds to quantum mechanics of a finite number of particles. Let $\Delta(S)$ be the space of formal linear combinations

$$
\Delta(S)=\left\{\sum^{\text {finite }} z_{k} W\left(f_{k}\right)\right\}
$$

where $z_{k} \in \mathbf{C}, f_{k} \in S$, and $W\left(f_{k}\right)$ being abstract symbols called the Weyl operators. Clearly $\Delta(S)$ is a complex linear space. The product of two Weyl operators is defined as

$$
W(f) W(g)=e^{-i \sigma(f, g) / 2} W(f+g),
$$

while the *-operation as $W(f)^{*}=W(-f)$, and they are next extended to $\Delta(S)$ by linearity (anti-linearity) respectively. The completion $\Delta_{1}(S)$ of $\Delta(S)$ with respect to the norm $\left\|\sum z_{k} W\left(f_{k}\right)\right\|_{1}=\sum\left|z_{k}\right|$ is a Banach *-algebra. We now define a new norm on $\Delta_{1}(S)$ by

$$
\|R\|=\sup _{\pi}\|\pi(R)\|, \quad R \in \Delta_{1}(S),
$$

where the supremum is taken over all nondegenerate representations $\pi$ of $\Delta_{1}(S)$ for which $\pi(W(\lambda f)), f \in S$, is continuous in $\lambda \in \mathbf{R}$ with respect to the $\sigma$-weak topology on $\mathrm{B}\left(\mathcal{H}_{\pi}\right)$. The completion of $\Delta(S)$ with respect to this norm is a $C^{*}$-algebra, say $\mathcal{W}(S)$, which we refer to as the $C^{*}$-algebra of the canonical commutation relations [13]. It is worth noting that such an algebra is simple.

3. Promeasures on locally convex topological vector spaces. Suppose $E$ is a locally convex topological vector space over $\mathbf{R}$, i.e. such that its topology is defined by a family of seminorms separating points. It is clear that the topology of a locally convex space is always Hausdorff. Such spaces appear naturally in physical applications as spaces of test functions like, for example, the space of smooth functions with compact support. By $E^{\prime}$ we denote the topological dual, and by $E^{*}$ the algebraic dual of the space $E$. Let $I$ be the set of all closed linear subspaces $V$ in $E$ such that $\operatorname{dim}(E / V)<\infty$, and let $p_{V}: E \rightarrow E / V$ be the canonical projection. We say that $V \leq W, V, W \in I$, if $W \subset V$. For any $V \leq W$ we define a surjective linear map $p_{V W}: E / W \rightarrow E / V$ by $p_{V W}\left(p_{W} f\right)=p_{V} f, f \in E$. Then $\left(E / V, p_{V W}, I\right)$ is a projective net of finite dimensional locally convex (and hence locally compact) topological vector spaces. The projective 
limit of this net is canonically isomorphic to the topological space $E^{\prime *}$ equipped with the $\sigma\left(E^{\prime *}, E^{\prime}\right)$-topology. Let $M(E / V)$ denote the set of all complex measures on $E / V$ with finite variations. $M(E / V)$, when equipped with the natural sum and multiplication by scalars, the multiplication given by convolution $*$, and the norm $\left\|\mu_{V}\right\|=\left|\mu_{V}\right|(E / V)$, is a Banach algebra. As a Banach space $M(E / V)$ is the dual space to $C_{0}(E / V)$, the Banach space of continuous functions on $E / V$ vanishing at infinity with the sup-norm. By definition, see [14], a promeasure on $E$ is an arbitrary projective net $\left(\mu_{V},\left(p_{V W}\right)_{*}, I\right)$, where $\mu_{V}$ is a positive finite measure on $E / V,\left(p_{V W}\right)_{*}: M(E / W) \rightarrow M(E / V)$ is the induced algebraic homomorphism, and $\left(p_{V W}\right)_{*}\left(\mu_{W}\right)=\mu_{V}$ for all $V \leq W$. It is worth noting that in general the projective limit $\lim _{\leftarrow} \mu_{V}$ may not exist on $E^{\prime *}$. However, if $\operatorname{dim} E<\infty$, then any promeasure on $E$ can be identified with a measure in an obvious way. To simplify notation we shall denote a promeasure by $\left(\mu_{V}\right)_{V \in I}$ or just by $\mu$, if there is no risk of confusion. Because for all $V \leq W$,

$$
\mu_{V}(E / V)=\left(p_{V W}\right)_{*} \mu_{W}(E / V)=\mu_{W}\left(p_{V W}^{-1}(E / V)\right)=\mu_{W}(E / W),
$$

we have $\left\|\mu_{V_{1}}\right\|=\left\|\mu_{V_{2}}\right\|$ for all $V_{1}, V_{2} \in I$. This common value uniquely associated with the promeasure $\mu=\left(\mu_{V}\right)_{V \in I}$, is called its total mass and will be denoted by $\|\mu\|$. If the total mass is equal to one, we shall say that $\left(\mu_{V}\right)_{V \in I}$ is a probability promeasure on $E$.

Suppose now that $\left(\mu_{V}\right)_{V \in I}$ and $\left(\nu_{V}\right)_{V \in I}$ are promeasures on $E$. Since

$$
\left(p_{V W}\right)_{*}\left(\mu_{W} * \nu_{W}\right)=\left(p_{V W}\right)_{*}\left(\mu_{W}\right) *\left(p_{V W}\right)_{*}\left(\nu_{W}\right)=\mu_{V} * \nu_{V}
$$

$\mu * \nu=\left(\mu_{V} * \nu_{V}\right)_{V \in I}$ is again a promeasure on $E$, which we shall call the convolution of promeasures $\left(\mu_{V}\right)_{V \in I}$ and $\left(\nu_{V}\right)_{V \in I}$. It is clear that convolution of probability promeasures is also a probability promeasure. If $T: E \rightarrow E$ is $\mathbf{R}$-linear and continuous, then for any $V \in I$ also $T^{-1}(V) \in I$ and so the linear operator

$$
T_{V}: E / T^{-1}(V) \rightarrow E / V, \quad T_{V}\left(p_{T^{-1}(V)} f\right)=p_{V}(T f),
$$

$f \in E$, is well defined. Moreover, it is injective. Let $\left(T_{V}\right)_{*}: M\left(E / T^{-1}(V)\right) \rightarrow M(E / V)$ be the induced homomorphism of measure algebras. Proofs of the following simple facts can be found in [15].

Proposition 1. Suppose $\left(\mu_{V}\right)_{V \in I}$ is a promeasure on E. Then $\nu=\left(\nu_{V}\right)_{V \in I}$, where $\nu_{V}=$ $\left(T_{V}\right)_{*}\left(\mu_{T^{-1}(V)}\right)$, is also a promeasure on $E$, which we shall denote by $T_{*}(\mu)$. Moreover, $\left\|T_{*}(\mu)\right\|=\|\mu\|$.

Suppose now that $x^{\prime} \in E^{\prime}$. If $\mu=\left(\mu_{V}\right)_{V \in I}$ is a promeasure on $E$, then $\mu_{x^{\prime}}=\left(x^{\prime}\right)_{*}(\mu)$ is a finite measure on $\mathbf{R}$. Hence

$$
\mathcal{F}(\mu)\left(x^{\prime}\right)=\int_{-\infty}^{\infty} e^{i t} \mu_{x^{\prime}}(d t),
$$

is a function on $E^{\prime}$ which we shall call the Fourier transform of the promeasure $\mu$. It is a positive definite function which is continuous on every finite dimensional subspace of $E^{\prime}$ [14]. Let us recall that $E^{\prime}$ as the topological dual space is equipped with the $\sigma\left(E^{\prime}, E\right)$-topology. 
Proposition 2. If $\mu=\left(\mu_{V}\right)_{V \in I}$ and $\nu=\left(\nu_{V}\right)_{V \in I}$ are promeasures on $E$, then for all $x^{\prime} \in E^{\prime}$,

$$
\mathcal{F}(\mu * \nu)\left(x^{\prime}\right)=\mathcal{F}(\mu)\left(x^{\prime}\right) \cdot \mathcal{F}(\nu)\left(x^{\prime}\right)
$$

Proposition 3. If $T: E \rightarrow E$ is $\mathbf{R}$-linear and continuous, then for any promeasure $\mu$ on $E, \mathcal{F}\left(T_{*}(\mu)\right)=\mathcal{F}(\mu) \circ T^{\prime}$, where $T^{\prime}: E^{\prime} \rightarrow E^{\prime}$ is the dual operator.

Combining Propositions 2 and 3 we obtain the following:

$$
\mathcal{F}\left(\mu *\left(T_{*} \nu\right)\right)=\mathcal{F}(\mu) \cdot\left(\mathcal{F}(\nu) \circ T^{\prime}\right) .
$$

4. Perturbed convolution semigroups of promeasures. Suppose that $\left(S_{t}\right)_{t \geq 0}$ is a semigroup of R-linear and continuous operators in $E$, i.e. $S_{0}=$ id and $S_{t} \circ S_{s}=S_{t+s}$ for all $s, t \geq 0$. Let $\mu_{t}=\left(\mu_{V}(t)\right)_{V \in I}, t \geq 0$, be a family of probability promeasures on $E$. We shall say that $\mu_{t}$ is an $S_{t}$-perturbed convolution semigroup if $\mu_{0}=\delta_{\overrightarrow{0}}$, where $\delta_{\overrightarrow{0}}$ denotes the point measure concentrated in the zero vector in $E$, and for all $s, t \geq 0$,

$$
\mu_{t} *\left[\left(S_{t}\right)_{*} \mu_{s}\right]=\mu_{s+t},
$$

i.e. for all $V \in I$ the following equality holds

$$
\mu_{V}(t) *\left[\left(S_{t, V}\right)_{*} \mu_{S_{t}^{-1}(V)}(s)\right]=\mu_{V}(s+t) .
$$

Let $r, s, t \geq 0$. The calculations

$$
\begin{gathered}
\mu_{t+s} *\left[\left(S_{t+s}\right)_{*} \mu_{r}\right]=\left[\mu_{t} *\left(S_{t}\right)_{*} \mu_{s}\right] *\left[\left(S_{t}\right)_{*}\left(S_{s}\right)_{*} \mu_{r}\right] \\
=\mu_{t} *\left[\left(S_{t}\right)_{*}\left(\mu_{s} *\left(S_{s}\right)_{*} \mu_{r}\right)\right]=\mu_{t} *\left[\left(S_{t}\right)_{*} \mu_{s+r}\right]
\end{gathered}
$$

show that this notion is well defined. Construction of perturbed semigroups is similar to that of typical convolution semigroups. For example, one can prove the following.

THEOREM 4. Suppose that $f_{0} \in E, Q$ is a quadratic positive form on $E^{\prime}, \nu$ is a probability promeasure on $E$ and $a>0$. Moreover, suppose that

a) $S_{t}^{\prime}: E^{\prime} \rightarrow E^{\prime}$ is locally $Q$ integrable, i.e. for any $x^{\prime} \in E^{\prime}$ and all $t>0$ the following integral

$$
Q_{t}\left(x^{\prime}\right)=\int_{0}^{t} Q\left(S_{r}^{\prime} x^{\prime}\right) d r \quad \text { exists }
$$

b) $t \rightarrow S_{t} f_{0}$ is weakly locally integrable,

c) $t \rightarrow\left(S_{t, V}\right)_{*} \nu_{S_{t}^{-1}(V)} \in M(E / V)$, where $S_{t, V}: E / S_{t}^{-1}(V) \rightarrow E / V$ is weakly*measurable for all $V \in I$.

Then the family $\mu_{t}, t \geq 0$, given by

$$
\mu_{t}=\mu_{t}^{D} * \mu_{t}^{Q} * \mu_{t}^{P}
$$

is an $S_{t}$-perturbed convolution semigroup on $E$, where

$$
\mu_{t}^{D}=\delta\left(\int_{0}^{t} S_{r} f_{0} d r\right), \quad \int_{0}^{t} S_{r} f_{0} d r \in E^{*},
$$


$\mu_{t}^{Q}$ is the Gaussian promeasure associated with $Q_{t}$, i.e. the unique promeasure on $E$ such that $\mathcal{F}\left(\mu_{t}^{Q}\right)\left(x^{\prime}\right)=e^{-Q_{t}\left(x^{\prime}\right) / 2}$, and

$$
\mu_{t}^{P}=e^{-a t} \exp \left[a \int_{0}^{t}\left(S_{r}\right)_{*} \nu d r\right]
$$

is the Poisson promeasure associated with the promeasure $\int_{0}^{t}\left(S_{r}\right)_{*} \nu d r$.

Proof. Because the convolution of $S_{t}$-perturbed convolution semigroups is also an $S_{t^{-}}$ perturbed convolution semigroup, it is enough to prove that each factor in equation (4) is an $S_{t}$-perturbed convolution semigroup. We check this property for the last case, i.e. for $\mu_{t}^{P}$. To this end we proceed by steps.

Step 1. Because the function $t \rightarrow\left(S_{t, V}\right)_{*} \nu_{S_{t}^{-1}(V)}$ is weakly*-measurable and $\left\|\left(S_{t, V}\right)_{*} \nu_{S_{t}^{-1}(V)}\right\|=1$ for all $t \geq 0$, there exists a measure $\nu_{V}(t) \in M(E / V)$ such that for any $f \in C_{0}^{t}(E / V)$,

$$
\nu_{V}(t)(f)=\int_{0}^{t}\left(S_{s, V}\right)_{*} \nu_{S_{s}^{-1}(V)}(f) d s .
$$

We show that $\nu_{t}=\left(\nu_{V}(t)\right)_{V \in I}$ is a promeasure on $E$. Let $V \leq W$. Then, for any $t \geq 0$,

$$
\begin{gathered}
\left(p_{V W}\right)_{*} \nu_{W}(t)=\left(p_{V W}\right)_{*}\left[\int_{0}^{t}\left(S_{s, W}\right)_{*} \nu_{S_{s}^{-1}(W)} d s\right]=\int_{0}^{t}\left(p_{V W} \circ S_{s, W}\right)_{*} \nu_{S_{s}^{-1}(W)} d s \\
=\int_{0}^{t}\left(S_{s, V} \circ p_{S_{s}^{-1}(V) S_{s}^{-1}(W)}\right)_{*} \nu_{S_{s}^{-1}(W)} d s=\int_{0}^{t}\left(S_{s, V}\right)_{*} \nu_{S_{s}^{-1}(V)} d s=\nu_{V}(t) .
\end{gathered}
$$

Step 2. If $\nu$ is a promeasure on $E$, then $\mu=\left(\mu_{V}\right)_{V \in I}$ given by $\mu_{V}=e^{\nu_{V}}$ is also a promeasure on E. By definition,

$$
\mu_{V}=\delta_{\overrightarrow{0}}+\nu_{V}+\frac{\nu_{V} * \nu_{V}}{2 !}+\ldots
$$

Because $\left\|\nu_{V} * \nu_{V}\right\|=\left\|\nu_{V}\right\|^{2}$, the series is norm convergent in $M(E / V)$. Suppose that $V \leq W$. Because $\left(p_{V W}\right)_{*}$ is norm continuous,

$$
\left(p_{V W}\right)_{*} \mu_{W}=\lim _{n \rightarrow \infty}\left(p_{V W}\right)_{*} \sum_{k=0}^{n} \frac{\left(\nu_{W}\right)^{k}}{k !}=\mu_{V} .
$$

Step 3. For a probability promeasure $\nu$ on $E$ we define

$$
\mu_{t}^{P}=e^{-a t} \exp \left[a \int_{0}^{t}\left(S_{s}\right)_{*} \nu d s\right],
$$

where $a>0$. By steps 1 and $2, \mu_{t}^{P}$ is a probability promeasure on $E$ such that $\mu_{0}^{P}=\delta_{\overrightarrow{0}}$. We show that $\left(\mu_{t}^{P}\right)_{t \geq 0}$ is an $S_{t}$-perturbed convolution semigroup. Let $V \in I$. Then (we omit the upper index $P$ )

$$
\begin{gathered}
\mu_{V}(t) *\left[\left(S_{t, V}\right)_{*} \mu_{S_{t}^{-1}(V)}(s)\right]=e^{-a(t+s)} \exp \left[a\left(\nu_{V}(t)+\left(S_{t, V}\right)_{*} \nu_{S_{t}^{-1}(V)}(s)\right)\right] \\
=e^{-a(t+s)} \exp \left[a\left(\int_{0}^{t}\left(S_{r, V}\right)_{*} \nu_{S_{r}^{-1}(V)} d r+\int_{0}^{s}\left(S_{t, V}\right)_{*}\left(S_{r, S_{t}^{-1}(V)}\right)_{*} \nu_{S_{r}^{-1}\left(S_{t}^{-1} V\right)} d r\right)\right] .
\end{gathered}
$$

Because $S_{t, V} \circ S_{r, S_{t}^{-1}(V)}=S_{r+t, V}$, we have

$$
\mu_{V}(t) *\left[\left(S_{t, V}\right)_{*} \mu_{S_{t}^{-1}(V)}(s)\right]
$$




$$
\begin{gathered}
=e^{-a(t+s)} \exp \left[a\left(\int_{0}^{t}\left(S_{r, V}\right)_{*} \nu_{S_{r}^{-1}(V)} d r+\int_{0}^{s}\left(S_{t+r, V}\right)_{*} \nu_{S_{t+r}^{-1}(V)} d r\right)\right] \\
=e^{-a(t+s)} \exp \left[a\left(\int_{0}^{t+s}\left(S_{r, V}\right)_{*} \nu_{S_{r}^{-1}(V)} d r\right)\right]=\mu_{V}(t+s) .
\end{gathered}
$$

Guided by the theory of stochastic processes we shall call such a semigroup the Poisson $S_{t}$-perturbed convolution semigroup.

5. Stochastic dynamics on CCR algebras. In order to construct a quantum dynamical semigroup on $\mathcal{W}=\mathcal{W}(S)$ we combine a deterministic evolution given by a semigroup of injective algebraic homomorphisms of $\mathcal{W}$ and a stochastic evolution represented by a perturbed convolution semigroup of probability promeasures. Let $\left(S_{t}\right)_{t \geq 0}$ be a semigroup of R-linear and continuous operators on $(S, \tau)$ such that $\sigma\left(S_{t} f, S_{t} g\right)=\sigma(f, g)$ for all $f, g \in S$ and all $t \geq 0$. It was shown in [16] that with such a semigroup one can associate a semigroup of algebraic and unital *-homomorphisms $\alpha_{t}: \mathcal{W} \rightarrow \mathcal{W}$ which extend the maps $\alpha_{t}(W(f))=W\left(S_{t} f\right), f \in S$. It is worth pointing out that since $\mathcal{W}$ is simple, all $\alpha_{t}$ are injective. It is clear that such a semigroup generalizes the notion of automorphic evolution. Since all $\alpha_{t}$ are injective and map unitary operators from $\mathcal{W}$ into unitary operators we shall say that $(\alpha)_{t \geq 0}$ represents a deterministic evolution of the system.

Now let $E=S^{\prime}$, where $S^{\prime}$ is the topological dual space to $(S, \tau) . S^{\prime}$ with the $\sigma\left(S^{\prime}, S\right)$ topology is a locally convex topological vector space over $\mathbf{R}$ such that $S^{\prime \prime}=S$. Since the topology $\tau$ is stronger than the norm topology, the inclusion $S \subset \mathcal{H} \subset S^{\prime}$ holds, where $\mathcal{H}=\bar{S}$, see sec. 2. Let $\left(S_{t}^{\prime}\right)_{t \geq 0}, S_{t}^{\prime}: S^{\prime} \rightarrow S^{\prime}$, the dual semigroup. By definition, $S_{t}^{\prime}$ are R-linear and continuous operators on $S^{\prime}$ such that $\left(S_{t}^{\prime}\right)^{\prime}=S_{t}$.

THEOREM 5. Suppose that $\left(\mu_{t}\right)_{t \geq 0}$ is an $S_{t}^{\prime}$-perturbed convolution semigroup of probability promeasures on $S^{\prime}$. Then there exists a unique quantum dynamical semigroup $\left(T_{t}\right)_{t \geq 0}$, $T_{t}: \mathcal{W} \rightarrow \mathcal{W}$, such that

$$
T_{t} W(f)=\mathcal{F}\left(\mu_{t}\right)(f) W\left(S_{t} f\right)
$$

for all $f \in S$.

Proof. Let $T_{t}^{0} W(f)=\Gamma_{t}(f) W(f)$, where $\Gamma(f)=\mathcal{F}\left(\mu_{t}\right)(f)$, and $f \in S$. We show that the operator $T_{t}^{0}$ can be extended to a completely positive norm contractive and unital operator on $\mathcal{W}$. By linearity, $T_{t}^{0}: \Delta(S) \rightarrow \Delta(S)$. The space $S$ when equipped with the discrete topology is an Abelian group whose dual group (the group of characters) $\hat{S}$ equipped with the Gelfand topology is a commutative compact group. The pairing between $S$ and $\hat{S}$ we denote by $(f, \hat{f}), f \in S$ and $\hat{f} \in \hat{S}$. Let us recall that the Gelfand topology on $\hat{S}$ is defined by the system of neighborhoods of the neutral element

$$
\left\{O\left(f_{1}, \ldots, f_{n} ; r\right): r>0, n \in \mathbf{N}, f_{i} \in S\right\},
$$

where

$$
O\left(f_{1}, \ldots, f_{n}: r\right)=\left\{\hat{f} \in \hat{S}:\left(f_{i}, \hat{f}\right) \in U_{r} \forall i=1, \ldots, n\right\},
$$

and $U_{r}=\{z \in \mathbf{C}:|1-z|<r\}$. With a character $\hat{f}$ one can associate a *automorphism $\beta_{\hat{f}}$ of $\Delta(S)$ defined by $\beta_{\hat{f}} W(f)=(f, \hat{f}) W(f)$, and then extended by linearity to $\Delta(S)$. 
Since $\Gamma_{t}$ is a positive definite function on the group $S$ and $\Gamma_{t}(\overrightarrow{0})=1$, for any $t \geq 0$ there exists a probability Borel measure $\hat{\mu}_{t}$ on $\hat{S}$ such that

$$
\Gamma_{t}(f)=\int_{\hat{S}}(f, \hat{f}) \hat{\mu}_{t}(d \hat{f}),
$$

see for example [17]. Hence, for any $x \in \Delta(S)$,

$$
T_{t}^{0}(x)=\int_{\hat{S}} \beta_{\hat{f}}(x) \hat{\mu}_{t}(d \hat{f}) .
$$

Because $\left\|\beta_{\hat{f}}(x)\right\|=\|x\|$, we have $\left\|T_{t}^{0}(x)\right\| \leq\|x\|$, and $T_{t}^{0}$ can be extended to a contractive operator on the algebra $\mathcal{W}$. It is also clear that $T_{t}^{0}(\mathbf{1})=\mathbf{1}$. Since the formula $(6)$ holds for any $A \in \mathcal{W}$, for all $A_{1}, \ldots, A_{n}$ and $B_{1}, \ldots, B_{n}$ from $\mathcal{W}$ we get

$$
\begin{gathered}
\sum_{i, j=1}^{n} B_{j}^{*}\left(T_{t}^{0}\left(A_{j}^{*} A_{i}\right)\right) B_{i}=\sum_{i, j=1}^{n} B_{j}^{*}\left[\int_{\hat{S}} \beta_{\hat{f}}\left(A_{j}\right)^{*} \beta_{\hat{f}}\left(A_{i}\right) \hat{\mu}_{t}(d \hat{f})\right] B_{i} \\
=\int_{\hat{S}}\left(\sum_{j=1}^{n} \beta_{\hat{f}}\left(A_{j}\right) B_{j}\right)^{*}\left(\sum_{i=1}^{n} \beta_{\hat{f}}\left(A_{i}\right) B_{i}\right) \hat{\mu}_{t}(d \hat{f}) \geq 0 .
\end{gathered}
$$

Thus $T_{t}^{0}$ is completely positive for all $t \geq 0$.

Let us now define $T_{t}: \mathcal{W} \rightarrow \mathcal{W}, T_{t}=\alpha_{t} \circ T_{t}^{0}, t \geq 0$. By definition, $T_{t}$ is a norm contractive completely positive and unital operator on $\mathcal{W}$. Let us check that $\left(T_{t}\right)_{t \geq 0}$ is a semigroup. Clearly, it is enough to show the semigroup property on Weyl operators. Suppose that $s, t \geq 0$ and $f \in S$. Then

$$
\begin{gathered}
\left(T_{s} \circ T_{t}\right) W(f)=\left(\alpha_{s} \circ T_{s}^{0}\right)\left(\alpha_{t} \circ T_{t}^{0}\right) W(f)=\left(\alpha_{s} \circ T_{s}^{0}\right)\left(\Gamma_{t}(f) W\left(S_{t} f\right)\right) \\
=\Gamma_{t}(f) \Gamma_{s}\left(S_{t} f\right) W\left(S_{s+t} f\right) .
\end{gathered}
$$

Because $\left(\mu_{t}\right)_{t \geq 0}$ is an $S_{t}^{\prime}$-perturbed convolution semigroup on $S^{\prime}$, by formula (1) we have $\Gamma_{t}(f) \Gamma_{s}\left(S_{t} f\right)=\Gamma_{s+t}(f)$. Hence $\left(T_{s} \circ T_{t}\right) W(f)=T_{s+t} W(f)$.

6. Extension of $T_{t}$ to $\pi(\mathcal{W})^{\prime \prime}$. Suppose that $S_{t}=e^{i t H}, H=H^{*}: \bar{S} \rightarrow \bar{S}$, and $H: S \rightarrow S$.

REMARK. For a regular representation $\pi$ of $\mathcal{W}$ the mapping $\pi(W(f)) \rightarrow \pi\left(W\left(e^{i t H} f\right)\right)$ extends to a one-parameter group of $\pi$-inner automorphisms of $\pi(\mathcal{W})^{\prime \prime}$.

For any $t \geq 0$ and $f \in S$

$$
\mathcal{F}\left(\mu_{t}\right)(f)=\int_{\hat{S}}(f, \hat{f}) \hat{\mu}_{t}(d \hat{f}),
$$

where $\hat{\mu}_{t}$ is a probability Borel measure on $\hat{S}$. For any $\hat{f} \in \hat{S}$ there exists an automorphism $\alpha_{\hat{f}}$ of $\mathcal{W}$ given by $\alpha_{\hat{f}} W(f)=(f, \hat{f}) W(f)$. The automorphism $\alpha_{\hat{f}}$ of $\mathcal{W}$ is called $\pi$ extendable if there exists an automorphism $\bar{\alpha}_{\hat{f}}: \pi(\mathcal{W})^{\prime \prime} \rightarrow \pi(\mathcal{W})^{\prime \prime}$ such that

$$
\left.\bar{\alpha}_{\hat{f}}\right|_{\pi(\mathcal{W})}=\pi \circ \alpha_{\hat{f}} \circ \pi^{-1}
$$

From the very definition one can show the following. 
THEOREM 6. If for any $t>0$ there exists a Borel set $B_{t} \subset \hat{S}$ such that

a) $\forall \hat{f} \in B_{t} \quad \alpha_{\hat{f}}$ is $\pi$-extendable,

b) $\hat{\mu}_{t}\left(B_{t}\right)=1 \forall t>0$,

then $\pi \circ T_{t} \circ \pi^{-1}: \pi(\mathcal{W}) \rightarrow \pi(\mathcal{W})$ has a unique extension to a quantum Markov semigroup on $\pi(\mathcal{W})^{\prime \prime}$.

REMARK. Automorphisms associated to characters $f \rightarrow e^{i \sigma(g, f)}$ are $\pi$-extendable for all $\pi$.

Acknowledgements. The paper has been supported by the Polish Ministry of Scientific Research and Information Technology under the grant No PBZ-MIN-008/P03/2003.

\section{References}

[1] D. F. Walls and G. J. Milburn, Quantum Optics, Springer, Berlin, 1994.

[2] W. H. Zurek, Phys. Rev. D 26 (1982), 1862.

[3] E. Joos and H. D. Zeh, Z. Phys. B 59 (1985), 223.

[4] R. Olkiewicz, Commun. Math. Phys. 208 (1999), 245.

[5] R. Olkiewicz, Annals Phys. 286 (2000), 10.

[6] R. Omnes, Phys. Rev. A 65 (2002), 052119.

[7] A. Majewski and B. Zegarliński, Markov Proc. and Rel. Fields 2 (1996), 87.

[8] A. Majewski, R. Olkiewicz and B. Zegarliński, J. Phys. A 31 (1998), 2045.

[9] Ph. Blanchard, P. Ługiewicz and R. Olkiewicz, Phys. Lett. A 314 (2003), 29.

[10] B. Demoen, P. Vanheuverzwijn and A. Verbeure, Lett. Math. Phys. 2 (1977), 161.

[11] B. Demoen, P. Vanheuverzwijn and A. Verbeure, Rep. Math. Phys. 15 (1979), 27.

[12] O. Bratteli and D. W. Robinson, Operator Algebras and Quantum Statistical Mechanics 2, Springer, Berlin, 1997.

[13] G. G. Emch, Algebraic Methods in Statistical Mechanics and Quantum Field Theory, Wiley Interscience, New York, 1972.

[14] N. Bourbaki, Eléments de mathématique. Intégration, Chapitre IX, Hermann, Paris, 1969.

[15] L. Schwartz, Radon Measures on Arbitrary Topological Spaces and Cylindrical Measures, Oxford University Press, 1973.

[16] J. Manuceau, Ann. Inst. Henri Poincaré 8 (1968), 139.

[17] W. Rudin, Fourier Analysis on Groups, Interscience Publ., New York, 1962. 